\title{
Occlusal caries detection in primary teeth: a comparison of DIAGNOdent with conventional methods
}

\author{
D. C. Attrill, ' and P. F. Ashley, ${ }^{2}$
}

\begin{abstract}
Objective To compare the accuracy and repeatability of three diagnostic systems (DIAGNOdent, visual and radiographic) for occlusal caries diagnosis in primary molars.

Design Two examiner, in vitro, blinded study. Histological gold standard.

Materials and methods 58 occlusal surfaces of primary molars examined in turn by two examiners using each of three diagnostic systems (DIAGNOdent, visual and radiographic). These results were compared with a histological gold standard. Sensitivity and specificity were calculated for each diagnostic system at a range of thresholds. Inter- and intra- examiner repeatability were calculated for each diagnostic system using the kappa statistic.

Main outcome measures Sensitivity, specificity, inter and intra examiner repeatability for each diagnostic system.

Results The highest sensitivity values were provided by DIAGNOdent ( 0.77 and 0.80 , examiners 1 and 2 respectively) however this was offset by a lower specificity $(0.82$ and 0.85$)$ than all other systems with the exception of examiner 1 at $\mathrm{V} 1$ visual threshold. The DIAGNOdent gave the highest values of kappa for intra- and inter- examiner repeatability with the exception of intra-examiner repeatability for examiner 2 where visual diagnosis had the highest value of kappa.

Conclusion The DIAGNOdent was the most accurate system tested for the detection of occlusal dentine caries in primary molars. The performance of the DIAGNOdent systems was not statistically significantly better than that achieved using visual examination for non-cavitated teeth (V1 and V2 threshold). DIAGNOdent may prove useful as a predictive clinical tool, however with appropriate training visual examination may offer similar results without the need for additional equipment.
\end{abstract}

T $\mathrm{t}$ is well established that caries levels in industrialised nations have decreased over the last few decades, available data also indicates that the greatest reductions have occurred on the smooth and approximal surfaces. As a result, occlusal surfaces are now perceived as being at the greatest risk of carious attack. Accompanying this change has been a shift in treatment philosophy; the original maxim of 'extension for prevention' has been eschewed for a minimal intervention approach, though this approach is only effective if caries is diagnosed at an early stage.

These changes have led to an increase in research and development

${ }^{1}$ Lecturer and Honorary Specialist Registrar, Unit of Prosthodontics, University of Manchester, Higher Cambridge Street, Manchester M15 6FH; ${ }^{2}$ Lecturer and

Honorary Specialist Registrar, Department of Paediatric Dentistry, Eastman

Dental Institute, 256 Gray's Inn Rd, London WC1X 8LD

${ }^{*}$ Correspondence to: $D$. C Attrill

email:david.attrill@man.ac.uk

REFEREED PAPER

Received 19.11.99; Accepted 06.12.00

(c) British Dental Journal 2001; 190: 440-443 of novel systems for the diagnosis of early occlusal caries. One such system is the DIAGNOdent (KaVo, Biberach/Riß, Germany). This illuminates the tooth surface with pulses of red laser light and analyzes the emitted fluorescence. Changes in the mineral content and porosity of the tooth surface result in a change in the pattern of fluorescence. The nature of emitted fluorescence correlates with the degree of demineralisation in the tooth and can be quantified a process known as quantitative laser fluorescence (QLF). A numerical value is assigned to the degree of fluorescence, which may be used as an indicator of the extent of caries.

Published evidence suggests that this new system has the ability to detect early occlusal caries in permanent teeth with greater accuracy and repeatability than conventional systems, such as bitewing radiography or visual diagnosis. ${ }^{1}$ However this evidence is derived from studies carried out in permanent teeth; there is no available data reporting its effectiveness on primary teeth. This is unfortunate as individuals with primary teeth, children, will benefit most from early caries diagnosis. Early caries detection in primary teeth is of great importance because of the rapid rate of caries progression that results from the reduced enamel thickness of primary teeth. ${ }^{2}$ In addition, one of the main barriers to treatment of the younger patient is fear. Early detection of caries in primary teeth would allow greater use of simple adhesive restorations or directed prevention techniques (eg professional fluoride application). Small carious lesions may not require local anaesthetic for caries removal, further removing patient barriers to treatment.

It could be argued that the results from permanent teeth could be extrapolated to primary teeth, however they have different morphological, anatomical and physiological characteristics. ${ }^{2}$ Therefore, the aims of this study were to:

1. Determine the accuracy of a new diagnostic system for occlusal caries diagnosis (DIAGNOdent) in primary molars when compared with a histological gold standard, and

2. Compare its accuracy with conventional diagnostic systems when used on primary teeth.

\section{Materials and methods}

\section{Introduction}

Sixty first and second primary molars extracted by an out-patient general anaesthetic clinic were selected for this in vitro study. They were stored in $10 \%$ neutral buffered formalin immediately following extraction. Inclusion characteristics for teeth in this study were the apparent absence of:

1. Occlusal restorations

2. Occlusal fissure sealants

3. Hypoplastic pits

4. Frank occlusal cavitation resulting from carious attack.

Occlusal surfaces were cleaned with a pumice and water slurry to 
Table I Manufacturers selected cut-off points for the DIAGNOdent

Peak surface reading Suggested clinical interpretation

\section{DIAGNOdent}

The DIAGNOdent system was calibrated prior to each batch of examinations and a recalibration performed after every ten teeth tested to minimise calibration drift. Probe tip A (narrow) was selected for use on occlusal surfaces.

Each tooth was rinsed in distilled water to remove fixing solution and dried with compressed air for 5 seconds. The unit was zeroed on a non carious, unstained portion of the tooth. The probe tip was then tracked across the entire occlusal surface and the peak reading recorded.

The presence or absence of occlusal pit and fissure caries was determined using the manufacturers suggested cut-off points (Table I)

\section{Visual}

Each tooth was dried for at least 5 seconds with compressed air and examined under a standard dental operating light. The presence or absence of occlusa pit and fissure caries was recorded using the criteria described by Ekstrand et al.: 5

V0 No or slight change in enamel translucency after prolonged air drying

VI Opacity or discolouration hardly visible on the wet surface but distinctly visible after air drying

V2 Opacity or discolouration distinctly visible without air-drying

V3 Localised enamel breakdown in opaque or discoloured enamel and/or greyish discolouration from the underlying dentine

V4 Cavitation in opaque or discoloured enamel exposing the dentine

\section{Radiographic}

Four teeth were radiographed per standard intra-oral film (Kodak Ektaspeed, Kodak Ltd, Herts, UK) and were mounted such that the occlusal plane of each tooth was parallel to the $\mathrm{x}$-ray beam and perpendicular to the film or imaging plate. The exposure was taken from the buccal aspect.

The $\mathrm{x}$-ray machine (Densomat, Philips, Eindhoven, The Netherlands) was set at $65 \mathrm{kVp}$ and $10 \mathrm{~mA}$, with a focal point to film/imaging plate distance of $30 \mathrm{~cm}$. Exposure times were 0.22 second. Radiographs were developed consecutively using an automatic processor.

Radiographs were then viewed under standardised conditions using a lightbox with no magnification. The presence or absence of occlusal pit and fissure caries was recorded using the criteria described by Ekstrand et al..$^{5}$

R0 No radiolucency visible

RI Radiolucency visible in the enamel

R2 Radiolucency visible in the dentine but restricted to the outer third of the dentine

R3 Radiolucency extending to the middle third of the dentine

R4 Radiolucency in the pulpal third of the dentine

remove any debris, and rinsed thoroughly in sterile water prior to examination.

Two examiners participated in this study; both were experienced in caries diagnosis. No specific training was performed prior to the study. Teeth were examined by both examiners using each of three diagnostic systems, namely:

1. Visual

2. DIAGNOdent

3. Radiographic

The examination techniques are detailed in Figure 1.

Examinations were performed independently and in a random order by each examiner. A random sub-sample (approximately $25 \%$ ) were subsequently re-examined as part of a repeatability study. Both inter- and intra- examiner repeatability were determined. Sufficient time was left between each examination to ensure the examiners did not recall the previous results and were blinded to all previous scores.

\section{Histological examination}

After all the examinations had been completed each tooth was hemi-sectioned through the fissure pattern in a mesial to distal direction using a band saw $0.1 \mathrm{~mm}$ thick (EXAKT-Apparatebau GmGH, Norderstedt, Germany). Wet sections were viewed under a stereomicroscope by a single examiner at x 50 magnification and

\begin{tabular}{ll} 
Peak surface reading & Suggested clinical interpretation \\
\hline $0-9$ & Sound/early enamel caries \\
$10-17$ & Enamel caries \\
$18-99$ & Dentinal caries \\
\hline
\end{tabular}

evaluated for the presence or absence of caries extending into enamel or dentine. The examiner was not aware of the previous diagnostic test results.

Ten teeth were selected at random and re-examined blind to establish the reproducibility of the histological assessment.

\section{Statistical methodology}

DIAGNOdent readings were recoded into three groups: sound, enamel caries and dentinal caries using the cut-off points detailed in Table 1.

Sensitivities (proportion of true disease identified correctly) and specificities (proportion of true non-disease identified correctly) were calculated for each diagnostic system using the histological gold standard. Comparisons were made between groups by using the $t$-test for comparing two proportions using the normal approximation to the binomial at the $P=0.05$ level.

Repeatability for each system was assessed by calculating Cohen's kappa. ${ }^{3}$

\section{Results}

Two teeth were excluded after close examination revealed the presence of occlusal restorations. After histological examination, 30 teeth were shown to have occlusal dentine caries, 5 teeth had enamel caries and 23 were sound. Twelve teeth $(40 \%)$ scored as having dentine caries had lesions extending to the middle third of the dentine or deeper. These results are summarised in Figure 2.

DIAGNOdent readings ranged from 0 to 99 . After recoding, both examiner 1 and examiner 2 scored 23 surfaces as sound, 7 with enamel caries and 28 with dentine caries. The frequency distribution of scores for each examiner using visual and radiographic examination are illustrated in Figures 3 and 4. It can be seen that both examiners had a similar pattern of scoring.

Values of sensitivity and specificity for the detection of occlusal caries extending into dentine were calculated for the DIAGNOdent (examiner 1 , sensitivity $=0.77$, specificity $=0.82$; examiner 2 , sensitivity $=0.80$, specificity $=0.85)$. Sensitivities and specificities were also calculated for the visual and radiographic systems. In these cases each of the classifications (V1, V2...etc; R1, R2 ...etc.) was used as a cut-off point for the detection of dentinal caries. Data for the detection of enamel caries were not calculated because of the small numbers of teeth with enamel caries. These data are

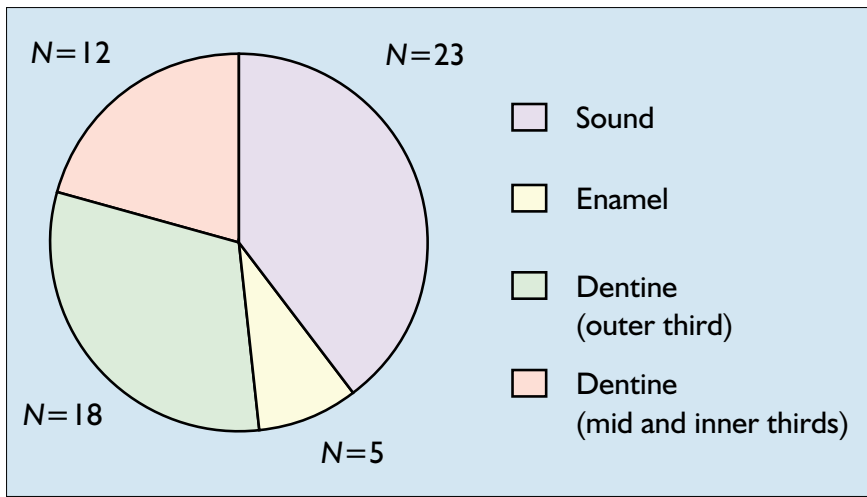

Fig. 2 Distribution of caries lesions (classified by depth) as determined by histological examination 


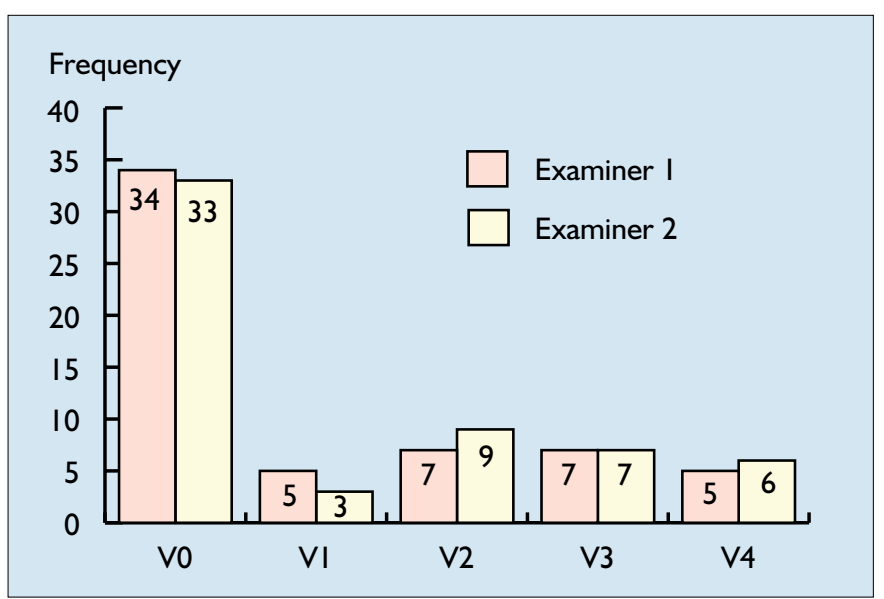

Fig. 3 Frequency distribution of visual scores for both examiners

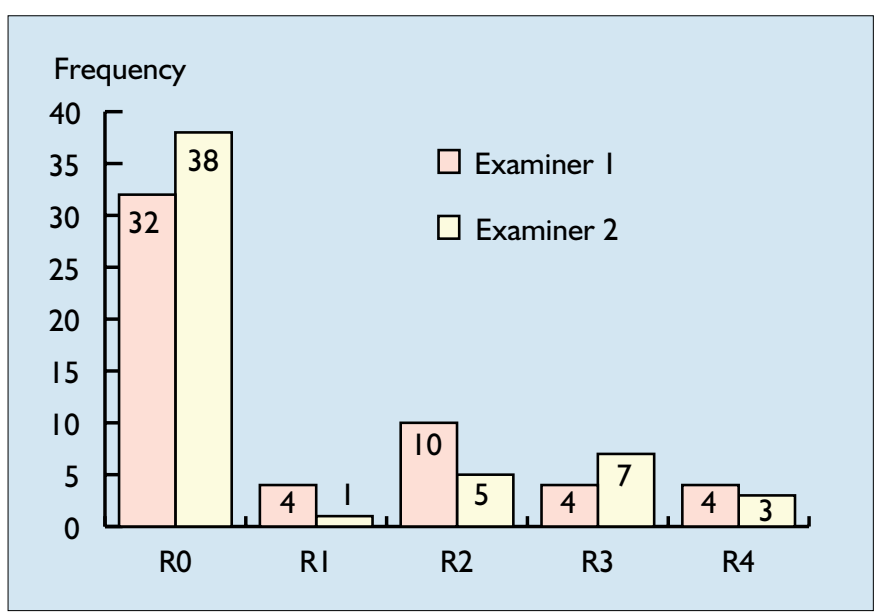

Fig. 4 Frequency distribution of radiographic scores for both examiners summarised in Table 2 .

Inter-examiner repeatability was calculated for the whole sample of 58 teeth. Intra-examiner repeatability was calculated using a $26 \%$ subgroup $(n=15)$ selected at random from the main group of samples and performed on a separate occasion from the main examination. This sub-group had a similar distribution of lesions as the main group based on the histological gold standard.

The kappa value for inter-examiner repeatability was highest for DIAGNOdent (0.7), and lowest for radiography (0.56). Examiner 1 was most repeatable with the DIAGNOdent for intra-examiner repeatability (0.78), and examiner 2 gave the best repeatability for visual diagnoses (0.77). These results are summarised in Table 3. The histological examination was shown to be $100 \%$ repeatable.

\section{Discussion}

The highest sensitivity values were provided by DIAGNOdent $(0.77$ and 0.80 examiners 1 and 2 respectively) however this was offset by a lower specificity $(0.82$ and 0.85$)$ than all other systems with the exception of examiner 1 specificity at $\mathrm{V} 1$ visual threshold.

Significant differences $(P<0.05)$ were demonstrated for sensitivity between DIAGNOdent and visual examination at V3 and V4 level (both examiners), and for specificity at V4 level (both examiners), with DIAGNOdent having higher sensitivity and lower specificity when compared with visual examination at these thresholds.

In comparisons between DIAGNOdent and radiography, sensitivity values were significantly different $(P<0.05)$ for examiner 2 at all levels, and for examiner 1 at the R3 and R4 threshold. Significant differences were also shown between the specificities at R2, R3 and $\mathrm{R} 4$ level (examiner 2) and R3 and R4 (examiner 1). As with visual examination, DIAGNOdent offered greater sensitivities and lower specificities at these thresholds.

The DIAGNOdent gave the highest values of kappa for intra- and inter- examiner repeatability with the exception of intra-examiner repeatability for examiner 2 , where visual diagnosis had the highest value of kappa. None of these kappa values were significantly different $(P>0.05)$ from any other.

The DIAGNOdent results are similar to those seen in the previous published study using permanent teeth, ${ }^{1}$ illustrating that this is an

Table 2 Sensitivities and specificities of visual, radiographic and DIAGNOdent classifications for the diagnosis of occlusal dentine caries (95\% confidence intervals in parentheses)

\begin{tabular}{|c|c|c|c|c|c|c|c|c|}
\hline & \multicolumn{4}{|c|}{ Sensitivity } & \multicolumn{4}{|c|}{ Specificity } \\
\hline & Examiner & & Examiner & & Examin & & Examine & \\
\hline $\begin{array}{l}\text { DIAGNOdent } \\
\text { VI } \\
\text { V2 } \\
\text { V3 } \\
\text { V4 } \\
\text { RI } \\
\text { R2 } \\
\text { R3 } \\
\text { R4 }\end{array}$ & $\begin{array}{l}0.77 \\
0.6 \\
0.57 \\
0.37^{\dagger} \\
0.17^{\dagger} \\
0.64 \\
0.57 \\
0.29^{\dagger} \\
0.14^{\dagger}\end{array}$ & $\begin{array}{l}(0.72,0.92) \\
(0.47,0.73) \\
(0.44,0.69) \\
(0.24,0.49) \\
(0.07,0.26) \\
(0.52,0.77) \\
(0.44 .0 .70) \\
(0.17,0.41) \\
(0.05,0.24)\end{array}$ & $\begin{array}{l}0.80 \\
0.7 \\
0.63 \\
0.4^{\dagger} \\
0.2^{\dagger} \\
0.54^{\dagger} \\
0.54^{\dagger} \\
0.36^{\dagger} \\
0.11^{\dagger}\end{array}$ & $\begin{array}{r}(0.7,0.9) \\
(0.58,0.82) \\
(0.51,0.76) \\
(0.27,0.53) \\
(0.1,0.3) \\
(0.4,0.67) \\
(0.4,0.67) \\
(0.23,0.48) \\
(0.02,0.19)\end{array}$ & $\begin{array}{l}0.82 \\
0.79 \\
0.93 \\
0.96 \\
I^{\dagger} \\
0.85 \\
0.92 \\
I^{\dagger} \\
I^{\dagger}\end{array}$ & $\begin{array}{r}(0.72,0.92) \\
(0.68,0.89) \\
(0.86,0.99) \\
(0.92,1) \\
\\
(0.75,0.94) \\
(0.85,0.99)\end{array}$ & $\begin{array}{l}0.85 \\
0.86 \\
0.89 \\
0.96 \\
I^{\dagger} \\
0.96 \\
I^{\dagger} \\
I^{\dagger} \\
I^{\dagger}\end{array}$ & $\begin{array}{r}(0.76,0.94) \\
(0.77,0.95) \\
(0.81,0.97) \\
(0.92,1) \\
(0.91,1)\end{array}$ \\
\hline
\end{tabular}

†Indicates statistically significant difference from DIAGNOdent $(P<0.05)$

Table 3 Kappa value of inter and intra-examiner repeatability for each diagnostic system (95\% confidence intervals in parenthesises)

\begin{tabular}{lrrrrrr}
\hline & \multicolumn{2}{c}{ Visual } & \multicolumn{2}{c}{ Radiographic } & DIAGNOdent \\
\hline & & & & & & \\
Examiner I & 0.50 & $(0.25,0.75)$ & 0.56 & $(0.23,0.88)$ & 0.78 & $(0.51,1)$ \\
Examiner 2 & 0.77 & $(0.42,1)$ & 0.60 & $(0.34,0.87)$ & 0.66 & $(0.36,0.96)$ \\
Examiner I vs 2 & 0.67 & $(0.51,0.82)$ & 0.56 & $(0.38,0.74)$ & 0.70 & $(0.54,0.86)$ \\
\hline
\end{tabular}




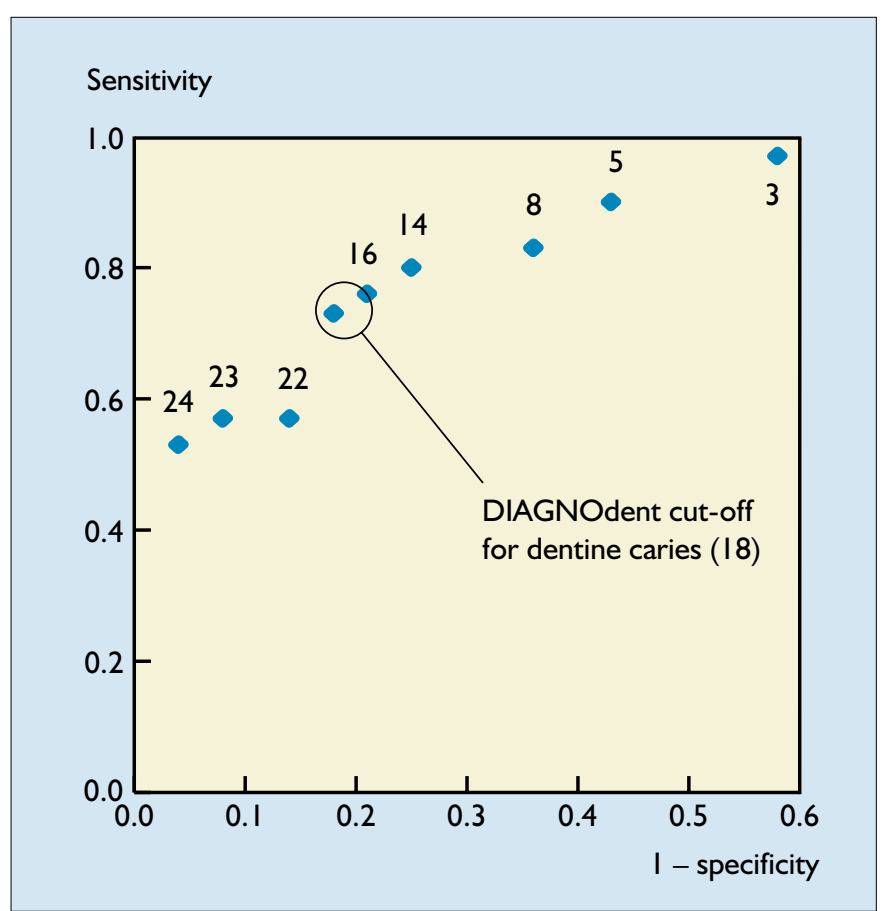

Fig. 5 Sensitivity vs I-specificity for DIAGNOdent dentine caries detection (examiner I) at different cut-off points (DIAGNOdent output values are indicated above each point)

effective instrument for the diagnosis of occlusal caries. The results for the visual technique were also similar to previously reported data on the diagnosis of occlusal caries in primary teeth ${ }^{4}$ (sensitivity $=0.45$, specificity $=1$ at the V3 diagnostic level). However previously reported data on permanent teeth ${ }^{5}$ using this particular grading system to describe the visual appearance of occlusal caries reported higher values for sensitivity and specificity. Differences between these studies may be caused by the different characteristics of primary and permanent teeth, however it is more likely that the differences reflect the examiner's lack of training in this method. In the previously reported study, ${ }^{5}$ the examiners were trained for 2 hours whilst in this study there was no specific training. The radiographic system was the worst performer in this study, this was not reflected by previously published data on primary teeth ${ }^{4}$ (sensitivity $=0.93$, specificity $=0.89$ at the $\mathrm{R} 2$ level). This difference is difficult to account for, however it does highlight the generally poor performance of bitewing radiography for the diagnosis of occlusal caries in primary teeth, particularly with early lesions.

The DIAGNOdent's performance is partially dependent on the cut-off points used to classify the numerical output into sound, enamel or dentine caries scores. The scale provided by the manufacturers may not be applicable for use with primary teeth. The effect of variations in the cut-off point can be illustrated by calculating the sensitivity and specificity for dentine caries diagnosis at each value of the DIAGNOdent reading and then plotting sensitivity $v s$ 1-specificity (ROC curve) as in Figure 5. As the cut-off point increases, so does the specificity, however this is at the expense of the sensitivity. When the sensitivity and specificity at each cut-off point for both examiners were compared, the manufacturer's cut-off point gave the highest combination. Therefore the cut-off point supplied with this instrument is suitable for use on primary teeth.

The visual system produced the highest combination of sensitivity and specificity at the V1/V2 levels (opacity or discolouration visible with or without air drying). This underlines the importance of the use of pre-cavitation diagnostic criteria. In addition it has implications for treatment, $50 \%$ of surfaces scored as V2 by examiner 2 had caries extending to the middle third of the dentine or deeper. It may be appropriate to recommend restoring these opaque/ discoloured areas with adhesive restorations after a minimal cavity preparation.

What is the clinical significance of these results? The experiment was carried out in vitro and therefore some care must be taken when extrapolating these results to the in vivo situation caused by the potential difficulties of examining children. Other experimental factors such as the effect of the storage medium (formalin) on the pattern of fluorescence are at this stage unquantifiable. Nevertheless these results can be used to give us an idea of the best system for occlusal caries diagnosis in primary teeth. In this instance, the best or most accurate system will be considered to be that system with the highest combination of sensitivity and specificity and highest value of kappa.

Bitewing radiography can be excluded from this comparison as it performed poorly overall when compared with the other two systems. This leaves the visual and DIAGNOdent systems, and it is difficult to differentiate between them. In this study the DIAGNOdent examination gave the highest overall combination of sensitivity and specificity, however these values were not significantly different $(P>0.05)$ from the visual examination if $\mathrm{V} 1$ or $\mathrm{V} 2$ was taken as the cut-off point for occlusal caries detection (examiners 1 and 2). The DIAGNOdent also gave consistently higher values for repeatability, again these were not significantly different $(P>0.05)$. In addition visual repeatability was calculated for each of the four possible cut-off points. If visual repeatability is recalculated, using only one cut-off point (V1) representing the presence or absence of dentine caries, then the kappa value should increase (the kappa value for inter-examiner repeatability rises to $0.75(95 \% \mathrm{CI}=0.58,0.92)$, for example).

In conclusion, the DIAGNOdent was the most accurate system tested for the detection of occlusal dentine caries in primary teeth. These differences were not statistically significant from the visual system at pre-cavitation levels (V1 and V2); DIAGNOdent may prove useful as a predictive clinical tool, however with appropriate training visual examination may offer similar results without the need for additional equipment. Conversely, when used by inexperienced examiners DIAGNOdent may be a useful adjunct to visual diagnosis by virtue of its accuracy and repeatability.

1 Lussi A, Imwinkelried S, Pitts N B, Longbottom C, Reich E. Performance and reproducibility of a laser fluorescence system for detection of occlusal caries in vitro. Caries Res 1999; 33: 261-266.

2 Mortimer K V. The relationship of deciduous enamel structure to dental disease. Caries Res 1970; 4: 206-223.

3 Cohen J. A coefficient of agreement for nominal scales. Educ Psychol Measurement 1960; 20: 37-46.

4 Ketley C E, Holt R D. Visual and radiographic diagnosis of occlusal caries in first permanent molars and in second primary molars. Br Dent J 1993; 174: 364-370.

5 Ekstrand K R, Ricketts D N J, Kidd E A M. Reproducibility and accuracy of three methods for assessment of demineralisation depth on the occlusal surface: an in vitro examination. Caries Res 1997; 31: 224-231. 